\title{
Soja - ein unterschätztes Pollen assoziiertes Nahrungsmittelallergen!?
}

\author{
Soy - an Underestimated Pollen Associated Food Allergen!?
}

Autoren

Institut
R. Treudler, S. Kramer, J.-C. Simon

Klinik für Dermatologie, Venerologie und Allergologie, Universitätsklinikum Leipzig A.ö.R.

\section{Bibliografie}

Dol $10.1055 / \mathrm{s}-0028-1119638$

Akt Dermatol 2009; 35:

145-150 @ Georg Thieme

Verlag KG Stuttgart · New York

ISSN 0340-2541

\section{Korrespondenzadresse \\ Priv.-Doz. Dr. med. habil. Regina Treudler}

Klinik für Dermatologie, Venerologie und Allergologie Universitätsklinikum Leipzig Philipp-Rosenthal-Str. 23 04103 Leipzig Regina.Treudler@ uniklinik-leipzig.de

\section{Zusammenfassung \\ 7}

Einhergehend mit einem steigenden Konsum wurden in den letzten Jahren in Deutschland zunehmend teils schwere allergische Reaktionen auf Sojaprodukte, insbesondere auf Sojagetränke, beobachtet. Birkenpollenallergiker haben ein besonderes Risiko, eine solche Reaktion zu erleiden, denn das Hauptallergen der Birke Bet v 1 zeigt eine hohe Homologie mit dem Soja-Allergen Gly $\mathrm{m} 4$. Hierdurch kann es bei entsprechend Sensibilisierten zu einer immunologischen Kreuzreaktion im Sinne einer Pollen assoziierten Nahrungsmittelallergie kommen. Genaue Daten zur Prävalenz dieses Krankheitsbildes sind nicht bekannt.
In Deutschland könnten allerdings bei geschätzten 5-10\% (4-8 Mio.) Birkenpollenallergikern bis zu 2,9 Mio. Menschen von einer Kreuzsensibilisierung auf Soja betroffen sein. Eine sichere Allergenmeidung ist schwierig, da Soja vielfach versteckt in Nahrungsmitteln enthalten sein kann. Besondere Hinweise für Birkenpollenallergiker, die insbesondere vor dem Verzehr proteinreicher, nicht-fermentierter Sojaprodukte (z.B. Drinks) gewarnt werden sollten, scheinen gerechtfertigt. Inwieweit auch durch eine spezifische Immuntherapie gegen Bet v 1 die Birken assoziierte Sojaallergie beeinflusst werden kann, ist nicht bekannt.

\section{Einleitung}

$\nabla$

Den Sojaprodukten werden eine Reihe von gesundheitsfördernden Eigenschaften zugeschrieben. Einhergehend mit einer entsprechenden Vermarktung als Lifestyle- und Wellnessprodukte hat der Konsum in den letzten Jahren zugenommen. Parallel dazu stellten sich bei Allergologen immer mehr Patienten vor, die nach Sojaverzehr über allergische Reaktionen, in der Regel vom Soforttyp, klagten [1,2]. Als besondere Risikogruppe konnten Birkenpollenallergiker identifiziert werden $[3,4]$.

In der folgenden Übersicht soll zunächst auf Soja als Nahrungsmittel eingegangen werden. Wie und wo kann dem Verbraucher dieses Allergen begegnen? Im Anschluss soll eine kurze Übersicht über den aktuellen Wissensstand zu Nahrungsmittelallergien im Allgemeinen und danach zu aktuellen Erkenntnissen zur Sojaallergie gegeben werden. Besondere Berücksichtigung findet dabei die Kreuzallergie zwischen dem Hauptallergen der Birke Bet v 1 und dem Soja-Allergen Gly $\mathrm{m}$ 4. Möglicherweise wird diese Kreuzallergie bisher unterschätzt, denn auch wenn keine ge- nauen Daten zur Prävalenz vorliegen, so könnten bis zu 2,9 Mio. Menschen in Deutschland von einer Birken assoziierten Sojaallergie betroffen sein.

\section{Soja als Nahrungsmittel \\ $\nabla$}

Die Sojabohne (Glycine maxima) gehört zur Familie der Hülsenfrüchte (Leguminosen). Neben Proteinen (32-42\%) und Fett (hoher Gehalt an nicht gesättigten Fettsäuren) enthalten Sojabohnen zahlreiche Mono-, Oligo- und Polysaccharide, Isoflavone, Phytoöstrogene sowie Ballaststoffe und Mineralien [5]. Die in einigen, vorwiegend asiatischen, Ländern beobachtete geringe Prävalenz an kardiovaskulären und bestimmten Tumor-Erkrankungen (z.B. Colon-, Mamma-, und Prostatakarzinom), hat man versucht mit dem Verzehr von Phytoöstrogenen und Isoflavonen aus der Sojabohne in Verbindung zu bringen [6]. So wird bis heute mit besonderem Hinweis auf diese vermuteten gesundheitsfördernden Eigenschaften intensiv für Sojaprodukte geworben. Die Evidenz für diese Indikationen bleibt jedoch 


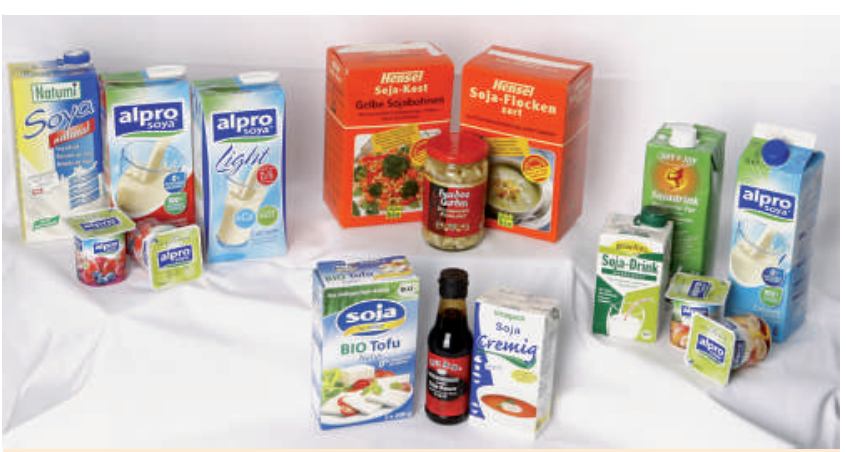

Abb. 1 Die Auswahl an Sojaprodukten zum Verzehr ist vielfältig.

Tab. 1 Vorkommen von Soja-Bestandteilen [8].

\begin{tabular}{|l|l|}
$\begin{array}{l}\text { Sojaprodukt } \\
\text { Vollfettprodukt }\end{array}$ & Nahrungs- bzw. Genussmittel \\
\hline $\begin{array}{l}\text { Puffsoja } \\
\text { Sojabohnen }\end{array}$ & $\begin{array}{l}\text { Süßwaren, Nährmittel } \\
\text { Back- und Süßwaren, Konserven }\end{array}$ \\
\hline Eiweißprodukte & $\begin{array}{l}\text { Fleischwaren, Fertiggerichte, Kinder- } \\
\text { nahrung, Backwaren, Suppen, Flocken }\end{array}$ \\
\hline Sojamehl & $\begin{array}{l}\text { Reformkost, Fertigmehle, Flocken, } \\
\text { Backwaren }\end{array}$ \\
\hline Sojakonzentrate & $\begin{array}{l}\text { Mixgetränke, Milchprodukte, Babykost, } \\
\text { Kaffeeweißer, Fleischprodukte }\end{array}$ \\
\hline Isoliertes Sojaeiweiß & $\begin{array}{l}\text { Margarine, Speisefette, Speiseöle, Salat- } \\
\text { saucen, Mayonnaise, Fischkonserven, } \\
\text { Ölprodukte }\end{array}$ \\
\hline Raffiniertes Sojaöl & Backwaren, Süßwaren, Fette, Salatsaucen \\
\hline Sojalecithin (Emulgator)
\end{tabular}

gering. Der Verbraucher kann sich einerseits bewusst für den Verzehr von Soja entscheiden, z.B. in Form von Sojagetränken, Sojabohnen, Sojajoghurt/-pudding, Tofu (Sojaquark), Miso (süße oder salzige Würzpaste), Natto (japanischer Sojakäse), Tempeh (Erzeugnis aus fermentierten Sojabohnen), Sauce ( $\bullet$ Abb. 1). Andererseits ist Soja auch versteckt - entweder als Zutat (zur Herstellung zugegeben) oder als Zusatzstoff (aus technologischen Gründen zugesetzt) - in Nahrungsmitteln enthalten, und der Verzehr findet oft unbewusst statt. So wird unter anderem Sojalecithin als Emulgator in Backmitteln und Süßwaren verwendet, Sojamehl oder -schrot zur Verbesserung von Brotteigen, Sojaprotein als Zusatz in Fleisch- und Wurstwaren und Sojavollmehl als Zusatz in Trockensuppen ( $\bullet$ Tab. 1).

\section{Nahrungsmittelallergien \\ $\nabla$}

Nahrungsmittelallergien treten häufig im Rahmen von Erkrankungen des atopischen Formenkreises auf. Als immunologisch vermittelte Reaktionen, meist IgE- oder zellulär vermittelt $[7,8]$, müssen sie von anderen Nahrungsmittel-Unverträglichkeiten abgegrenzt werden ( $\bullet$ Abb. 2).

Klinisch äußern sich Nahrungsmittelallergien einerseits mit Soforttyp-Reaktionen, z.B. in Form von oralem Allergie-Syndrom (OAS), Urtikaria/Angioödemen, Asthma, Magen-Darm- und Kreislaufbeschwerden. Aktuelle Daten aus Deutschland weisen darauf hin, dass Nahrungsmittel - bei Kindern sogar noch weit vor Insektengiften und Medikamenten - zu den häufigsten Auslösern der Anaphylaxie gehören $[9,10]$. Zudem kann es im Sinne einer Spättyp-Reaktion zur Verschlechterung eines atopischen Ekzems kommen. Die einzelnen Organe sollen durch IgE-vermittelte Nahrungsmittelallergien mit folgender Häufigkeit betroffen sein: Haut/Schleimhaut $45 \%$, Atemwege 25\%, Magen-DarmTrakt 20\%, Kreislauf 10\% [8].

Unter Bezug auf mögliche Sensibilisierungswege, betroffene Patienten und involvierte Allergene werden verschiedene Typen der Nahrungsmittelallergien abgegrenzt $[8,11]$ ( Tab.2): Die typischerweise im frühen Kindesalter erfolgende Typ A-Sensibilisierung erfolgt über den Verdauungstrakt.

Hier spielen hitze- und säurestabile Allergene wie z.B. Kuhmilch, Hühnerei und Erdnuss eine Rolle. Klinisch kann es zur Verschlechterung einer atopischen Dermatitis kommen, wobei sowohl IgE-vermittelte als auch zelluläre Reaktionen vorkommen. Zudem können - meist IgE-vermittelte - Soforttyp-Reaktionen bis hin zur Anaphylaxie auftreten [11].

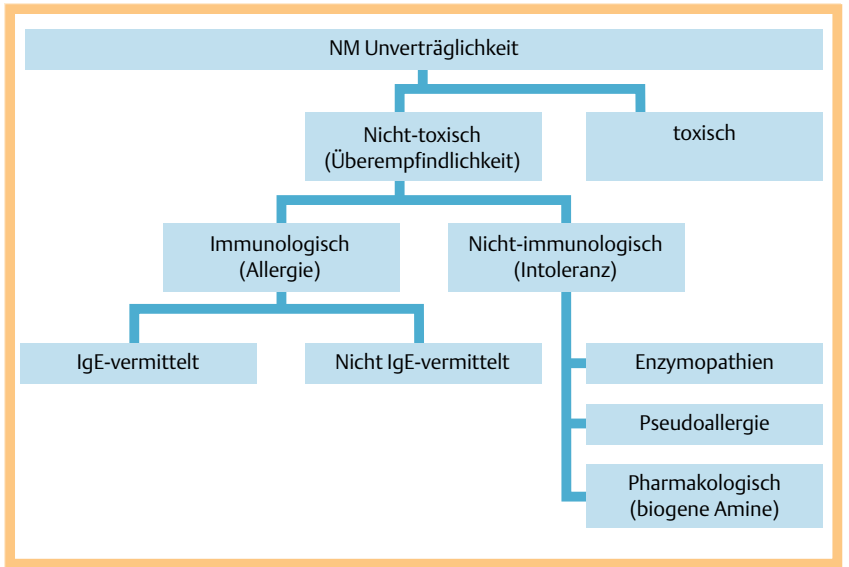

Abb. 2 Klassifikation von Nahrungsmittelunverträglichkeiten. Unter Nahrungsmittelallergien versteht man die immunologisch vermittelten Reaktionen [8].

\begin{tabular}{|c|c|c|c|}
\hline Sensibilisierungsweg & $\begin{array}{l}\text { Typ A (klassisch) } \\
\text { gastrointestinal }\end{array}$ & $\begin{array}{l}\text { Typ B (Kreuzreaktion) } \\
\text { inhalativ primär auf } \\
\text { Aeroallergene }\end{array}$ & $\begin{array}{l}\text { Typ C } \\
\text { gastrointestinal }\end{array}$ \\
\hline atopische Diathese & ja & ja & nein \\
\hline bevorzugtes Alter & Säuglinge/Kleinkinder & Schulalter/Erwachsene & Erwachsene (meist Frauen) \\
\hline $\begin{array}{l}\text { Eigenschaften } \\
\text { der Allergene }\end{array}$ & hitze- und säurestabil & $\begin{array}{l}\text { meist hitze- und säure- } \\
\text { labil, selten stabil }\end{array}$ & hitze- und säurestabil \\
\hline Beispiele & $\begin{array}{l}\text { Kuhmilch } \\
\text { Hühnerei } \\
\text { Erdnuss }\end{array}$ & $\begin{array}{l}\text { Kern-/Steinobst bei } \\
\text { Birkenpollenallergie }\end{array}$ & $\begin{array}{l}\text { Fisch } \\
\text { Meerestiere } \\
\text { Fleisch }\end{array}$ \\
\hline
\end{tabular}

Tab. 2 Nahrungsmittelallergien werden unter Bezug auf die Sensibilisierungswege, betroffene Patientengruppen sowie Eigenschaften der Allergene in mehrere Typen unterteilt (modifiziert nach $[8,11]$ ). 


\begin{tabular}{|c|c|c|c|c|}
\hline Sensibilisierung & Typische Betroffene & Exposition & Allergen & $\begin{array}{l}\text { Typische Klinik, } \\
\text { betroffene Organe }\end{array}$ \\
\hline \multirow[t]{4}{*}{ inhalativ } & Hafenarbeiter & inhalativ & Gly m 1 & Atemwege \\
\hline & & & Gly m 2 & \\
\hline & Pollenallergiker & gastrointestinal & Gly m 3 & OAS, Atemwege, GIT, \\
\hline & & & Gly m 4 & Kreislauf \\
\hline \multirow[t]{2}{*}{ gastrointestinal } & Kinder und & & Gly m 5 & GIT, auch andere \\
\hline & Erwachsene & & Gly m 6 & $\begin{array}{l}\text { Organe bevorzugt } \\
\text { schwere Reaktionen? }\end{array}$ \\
\hline
\end{tabular}

Tab. 3 Übersicht über bisher klassifizierte Allergene der Sojabohne. Diese unterscheiden sich bezüglich Sensibilisierungswegen, Betroffenen,

Exposition und typischer klinischer Manifestationen.
Ältere Kinder, Jugendliche und Erwachsene reagieren eher auf die dem Typ B zugeordneten - meist hitzelabilen - Allergene, bei denen die Sensibilisierung über die Atemwege erfolgt $[11,12]$. Hierbei handelt es sich in erster Linie um IgE-vermittelte Reaktionen auf Pollen assoziierte Nahrungsmittel. Bekannt sind insbesondere das Birken-Obst-, das Beifuß-Sellerie-Gewürz- sowie das Latex-Frucht-Syndrom, bei dem nach Verzehr der Nahrungsmittel meist ein OAS auftritt, welches gelegentlich auch eine Systembeteiligung bis hin zur Anaphylaxie zeigen kann. Bedeutsam ist, dass bei solchen kreuzallergisch bedingten TypB-Nahrungsmittelallergien bereits der Erstkontakt mit dem Nahrungsmittel zu Symptomen führen kann, ohne dass sich der Patient dieses Risikos bewusst ist [11,12]. Typ C entspricht einer Nahrungsmittelallergie bei Erwachsenen, i.d. R. ohne vorbestehende Atopie, die über den Gastrointestinaltrakt eine Sensibilisierung auf hitze- und verdauungsstabile Allergene erwerben (z.B. Fisch).

\section{Soja als Allergen}

Bisher werden mindestens 16 IgE-reaktive Proteine als Allergene in der Sojabohne vermutet, sechs Allergene sind aktuell klassifiziert [13-15] ( Tab. 3).

Davon zählen Gly m 1 und Gly m 2 zu den Soja-Hüll-Proteinen, die im Anschluss an eine primäre Sensibilisierung über die Atemwege zu einem Asthma führen können [16,17]. Die Sojaallergene Gly $\mathrm{m} 3$ und Gly $\mathrm{m} 4$ sind bei der sekundären Sojaallergie (Typ B-Nahrungsmittelallergie) involviert [16]. Bei Gly m 3 handelt es sich um ein Sojaprofilin. Gly m 4 ist ein 16,6 kDa großes Protein aus der Familie der Pathogenesis-Related-(PR)-10-Proteine. Es handelt sich hierbei um eine Proteinfamilie, die eine hohe Ähnlichkeit zum Birkenpollen-Majorallergen hat (sog. Bet v 1-Homologe) und zu denen auch z.B. das Apfelallergen Mal d 1 gehört [4,11]. Pflanzenbiologen beschrieben Anfang der 1990er-Jahre erstmals verschiedene Stress-induzierte Proteine, die gewebsspezifisch in bestimmten Entwicklungsphasen und auch bei Infektionen, Verletzungen oder auf Einwirkung anderer Stressstimuli in der Sojapflanze gebildet werden [18]. Einer dieser Vertreter war das sogenannte SAM-22 (starvation-associated message 22) [18]. Die Arbeiten von Kleine-Tebbe et al. führten dazu, dass SAM-22 als Bet v 1 kreuzreaktives Protein für zum Teil schwere allergische Reaktionen nach Sojagenuss identifiziert werden konnte [3,4]. 2002 wurde SAM-22 in Gly m 4 umbenannt und in die offizielle Allergen-Datenbank aufgenommen (www.allergen.org). Mittlerweile ist bekannt, dass ein dominantes T-Zell-Epitop von Bet v 1 eine Aminosäuresequenzähnlichkeit von $80 \%$ und eine Gesamt-Proteinähnlichkeit von $63 \%$ mit dem Gly m 4 aufweist [19]. Vor kurzem erst wurden die SojaSpeicherproteine $\beta$-Conglycin und Glycinin als Sojaallergene Gly $m 5$ und Gly m 6 klassifiziert [14]. Eine Sensibilisierung auf diese Proteine wurde bei 16 von 30 Patienten mit einer Sojaallergie gefunden und scheint mit dem Auftreten schwerer allergischer Reaktionen assoziiert zu sein. Eine Reihe weiterer Sojaproteine werden noch eingehender analysiert, u.a. Gly m Bd $28 \mathrm{k}$ (Speicherprotein), Gly m Bd 30k (Oil-body-associated protein), Trypsin-Inhibitor, 2S-Albumin [8,13,20].

\section{Klinische Manifestation der Sojaallergie}

Primäre (Typ A) Sojaallergien, die auf einer gastrointestinalen Sensibilisierung beruhen, sind selten und manifestieren sich vor allem bei Vorliegen einer atopischen Diathese bei Säuglingen und Kleinkindern, wenn diese aufgrund einer Kuhmilchallergie mit Sojaproteinformula ernährt werden [21]. Klinische Symptome dabei können Diarrhöen, Enterocolitis, Ekzemverschlechterung oder auch anaphylaktische Reaktion sein. Weitaus häufiger anzutreffen sind die Symptome einer Birken assoziierten Sojaallergie (Тур B) mit dem wesentlichen klinischen Bild des OAS. Hierbei kommt es zu lokaler Irritation, Juckreiz und Schwellungen der Lippen-, Mund- und Rachenschleimhaut. Bei einem Teil der Patienten kann es auch zu kutanen, respiratorischen, gastrointestinalen oder kardiovaskulären Begleiterscheinungen bis hin zum anaphylaktischen Schock kommen $[11,12]$.

\section{Håufigkeit einer Gly m 4-bedingten Sojaallergie} $\nabla$

Bisher liegen wenige Daten zur Prävalenz der Birken assoziierten Sojaallergie vor. Eine von uns durchgeführte erste Untersuchung in Leipzig ergab, dass von 50 konsekutiven Birkenpollenallergikern 46 (92\%) gegen Bet v 1 und 36 (72\%) gegen Gly m 4 sensibilisiert waren (Korrelation $(0,962$; p < 0.01) [1]. 28/50 Patienten gaben bei einer standardisierten Fragebogenuntersuchung an, bereits bewusst Sojaprodukte verzehrt zu haben (darunter Soja-Getränke $n=16$, Tofu $n=7$, Sojasauce $n=6$ ). Bemerkenswerterweise hatten 8/16 (50\%) der Patienten, die Sojagetränke konsumiert hatten, klinische Soforttypreaktionen erlitten. Kein einziger Patient hatte auf andere Sojaprodukte als auf die Getränke reagiert [1]. Mittlerweile konnten weiterführende Daten von 87 Birkenpollenallergikern aus Leipzig und Umgebung ausgewertet werden [22], von denen sich 50 an einen bewussten Sojakonsum erinnerten. 15/50 (30\%) gaben an, beim Verzehr Soforttypreaktionen entwickelt zu haben, wobei diese Reaktionen je nach verzehrtem Sojaprodukt unterschiedlich häufig auftraten und sich mit Abstand am häufigsten nach Drinks manifestierten. Die Tatsache, dass fast jeder zweite unserer Patienten (13/29;46\%) nach Sojadrinkverzehr anamnestisch eine Soforttypreaktion entwickelte [22], weist auf ein hohes Gefährdungspotenzial für Birkenpollenallergiker durch die schnelle Zufuhr großer Mengen gering verarbeiteten Sojaproteins hin. Klinisch waren neben 
dem OAS auch andere Organbeteiligungen bis hin zum Blutdruckabfall zu verzeichnen. ${ }^{1}$

Eine Studie aus der Schweiz [23] ergab, dass 71\% (67/94) der Patienten einer Gruppe von Birkenpollenallergikern mit hohen Bet v 1-Werten (> 17,5 kU/l) auch spezifisches IgE für Gly $m 4$ hatten. Allerdings gaben nur 9,6\% der in einem Telefoninterview Befragten an, Beschwerden nach dem Verzehr von Sojaprodukten bemerkt zu haben. Möglicherweise besitzen einige Birkenpollenallergiker kreuzreaktives IgE gegen Gly m 4, ohne klinisch relevante Reaktionen zu entwickeln. Andererseits könnten die im Vergleich zu unseren Daten deutlich niedrigeren Werte bezüglich der klinischen Relevanz auch daran liegen, dass die Befragten eventuell bisher noch keine Sojaprodukte mit hohem Gly m 4-Gehalt verzehrt haben (z. B. Getränke), so dass die die Zahl der Betroffenen dadurch unterschätzt werden würde. Genaue Daten zur Relevanz einer Gly m 4-Sensiblisierung sollten wünschenswerter Weise im Rahmen zukünftiger Studien durch eine orale Provokationstestung mit Sojaprotein gesichert werden. Geht man davon aus, dass 5-10\% der in Deutschland lebenden Menschen gegen Birkenpollen sensibilisiert sind (ca. 4-8 Mio. bei 80 Mio. Einwohnern), so sollten nach vorliegenden Daten ca. $90 \%$ gegen Bet v 1 (3,6 - 7,2 Mio.) und 70-80\% gleichzeitig gegen Gly $\mathrm{m} 4$ sensibilisiert sein (2,5-5,8 Mio.). 10-50\% könnten nach oben vorgestellten Daten eine klinisch relevante Sensibilisierung haben, entsprechend 0,25-2,9 Mio. Dieses entspräche einem Anteil von 0,3-3\% der Bevölkerung und läge somit womöglich höher als die aktuellen Schätzungen des Bundesinstitutes für Risikobewertung, die davon ausgehen, dass ca. 0,4\% der deutschen Bevölkerung an einer Sojaallergie leiden. Unter Berücksichtigung der Tatsache, dass Sojaprodukte auch immer mehr an Popularität im deutschsprachigen Raum gewinnen, ist mit einer Zunahme allergischer Reaktionen auf diese Produkte zu rechnen.

\section{Diagnostik der Sojaallergie \\ $\nabla$}

Bei Verdacht auf eine Sojaallergie sollte geklärt werden, ob eine Kreuzreaktion bei Bet v 1-Sensibilisierung vorliegt. Ein Prick-zuPrick-Test mit nativen, Gly m 4 haltigen Sojaprodukten (z. B. mit einem Sojagetränk) und die Bestimmung des spezifischen IgE gegen Gly $\mathrm{m} 4$ ist diagnostisch hilfreich. Kommerziell erhältliche Prick-Test-Extrakte aus der Sojabohne dagegen eignen sich aufgrund des geringen Gehalts an Gly m 4 nicht zur Diagnostik einer Sojaallergie $[1,2,24]$.

Auch in den kommerziell erhältlichen Sojaextrakten zur IgE-Bestimmung ist Gly $\mathrm{m} 4$ nur sehr schwach repräsentiert. In einer Studie von Lidholm et al. [25] reagierten nur 45\% der Sojaallergiker positiv auf den kommerziell erhältlichen Sojaextrakt, aber 96\% positiv auf das rekombinante Gly m 4 (Phadia, Uppsala, Schweden), das seit einigen Jahren auch für die klinische Routine verfügbar ist. Weitere Tests (z. B. Histamin- oder Leukotrienfreisetzungs- sowie Basophilenstimulationstests) können aufgrund mangelhafter Standardisierung und fehlender Validierung bisher nicht für die Routinediagnostik empfohlen werden. Eine Nahrungsmittelprovokationstestung kann bei unklaren Befunden zur Diagnosesicherung beitragen und wird insbesondere zur Bestimmung des Schwellenwertes an auslösendem Sojaprotein eingesetzt. Eine solche Untersuchung wurde in Form einer doppelblinden, Placebo kontrollierten Nahrungsmittelprovoka-

\footnotetext{
${ }^{1}$ Die Untersuchungen zur klinischen Relevanz einer Gly m 4 Sensibilisierung bei Birkenpollen-Allergikern in Leipzig sind Teil der Dissertationsarbeit von Frau cand. med. Susanne Kramer.
}

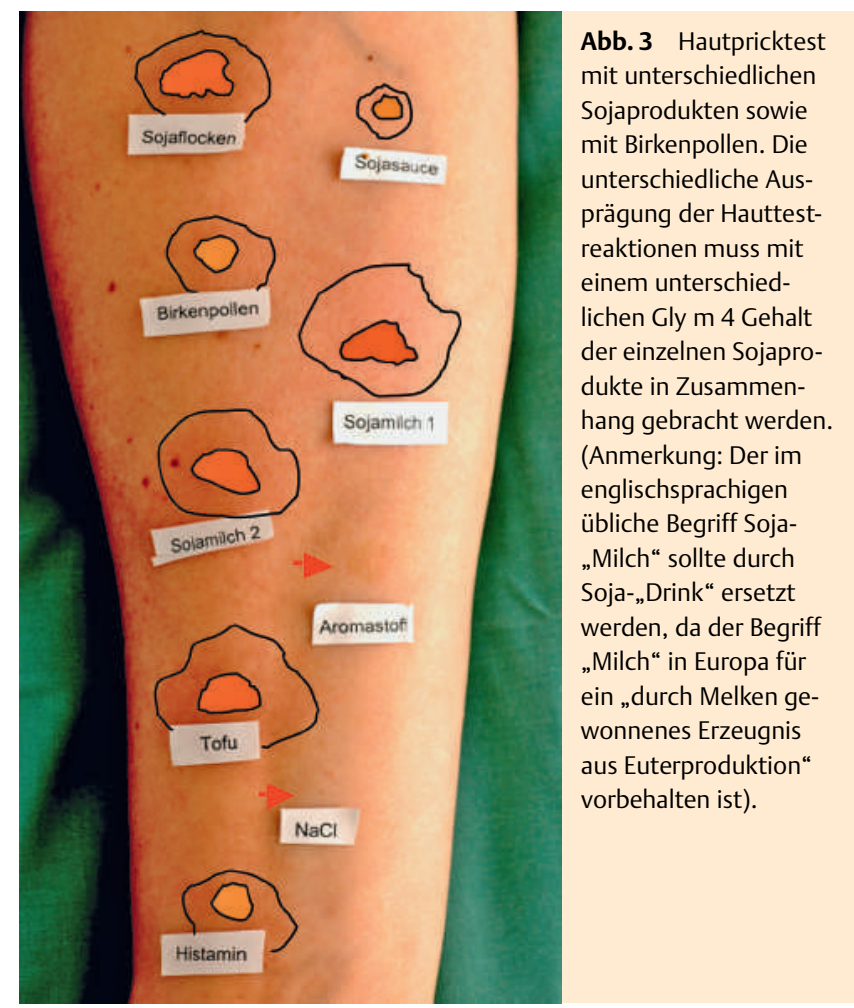

tion (DBPCFC) von Ballmer-Weber et al. [26] an 30 Soja-Allergikern vorgenommen. 21 von 30 (70\%) hatten spezifisches IgE gegen Bet v 1- und gegen Gly $\mathrm{m} 4$. Alle Patienten reagierten mindestens mit subjektiven Symptomen im Sinne eines OAS, 11 von 30 Patienten (37\%) entwickelten objektive Symptome, die bis zum Blutdruckabfall reichten. Die mittlere Schwellendosis zur Auslösung von Beschwerden lag für subjektive Symptome bei 5,8 (Spannweite 0,01 - 50) Gramm und für objektive Symptome bei 21,2 (0,454-50) Gramm Sojaprotein. Solche Proteinmengen können insbesondere durch den Verzehr eines Sojagetränkes schnell erreicht werden (z.B. sind in 100 Gramm eines häufig verzehrten Sojadrinks 3,3 Gramm Protein enthalten).

\section{Allergenität einzelner Sojaprodukte $\nabla$}

Die Konzentration an Gly m 4 erhöht sich mit der Zeit der Reifung und Lagerung der Sojabohnen und ist bei reifen Sojabohnen sechsmal höher als in unreifen. In einer Studie von Mittag et al. [23] wurden eine Anzahl von Sojaprodukten auf ihren Gehalt an Gly m 4 untersucht, wobei der höchste Gehalt in der rohen Sojabohne vorlag. Deutliche Mengen an Gly m 4 wurden in einem Sojaproteinpulver gefunden, etwas weniger aber doch in nennenswerte Konzentration in Sojadrinks, Sojaflocken und Tofu. In fermentierten Produkten wie Sojasauce, Miso und geröstete Sojabohnen konnte kein Gly m 4 nachgewiesen werden. Allerdings gibt es andere Berichte darüber, dass diese Produkte durchaus - je nach Verarbeitungsprozess - auch nennenswerte Gly m 4 Mengen enthalten können. Das Erhitzen der Sojaprodukte verringert offensichtlich den Gly $m$ 4-Gehalt nach und nach. Auch wenn das Gly $\mathrm{m} 4$ partiell hitzestabil zu sein scheint, so kann etwa nach $4 \mathrm{~h}$ Garzeit damit gerechnet werden, dass kein Gly m 4 mehr nachweisbar ist. Die unterschiedliche Allergenität einzelner Sojaprodukte kann auch durch unterschiedlich starke Reaktionen im Hautpricktest gezeigt werden ( $\bullet$ Abb. 3 ). 


\section{Deklarationspflicht und Karenzempfehlungen \\ $\nabla$}

Produkte mit großem Sojaproteingehalt, besonders in gering prozessierter Form, kommen prinzipiell als Auslöser von Gly m 4-bedingten Reaktionen in Frage. Der häufig schnelle Verzehr (z.B. in Form von Sojagetränken) und die rasche Verfügbarkeit der gelösten Proteine können zusätzliche Ursachen für die Häufigkeit und die Schwere der allergischen Reaktionen auf diese Produkte sein. Daher sollten besonders gering prozessierte Sojaprodukte, wie Sojagetränke, Sojajoghurt und -desserts, Sojaflocken, Diätpulver und Tofu von Birkenpollenallergikern gemieden werden. Stark fermentierte und erhitze Produkte, wie Sojasauce, Miso, Sojaöl und geröstete Sojabohnen stellen aufgrund ihres geringen Gly m 4-Gehalts meist keine große Gefahr für Allergiker dar. Dennoch sollten Betroffene versuchen, Produkte mit offensichtlichem Sojagehalt zu meiden. Eine systematische Vermeidung sämtlicher Nahrungsmittel, die kleinste Mengen von Sojaprotein enthalten, scheint jedoch aus allergologischer Sicht weder gerechtfertigt noch praktikabel [4].

Dass versteckte Nahrungsmittelallergene ein Problem für Sojaallergiker darstellen können, wurde kürzlich in einer retrospektiven Untersuchung an 436 Nahrungsmittelallergikern in Spanien gezeigt [27]: 119 (22.4\%) aller 530 angegebenen klinischen Reaktionen (bis hin zur Anaphylaxie) wurden durch versteckte Allergene und 13 von 31 (42\%) angegebenen Reaktionen auf Soja durch Nahrungsmittel mit versteckten Sojaproteinen induziert. Es handelte sich dabei um Sojaprotein in gekochtem Schinken, Würstchen, Käsegebäck, Fertiggerichten, Desserts und in Bratensauce [27].

Eine Allergenmeidung für Betroffene wurde ab November 2005 in der Europäischen Union dadurch erleichtert, dass eine Kennzeichnungspflicht für Lebensmittel in Kraft gesetzt wurde, die sich aktuell auf 14 Nahrungsmittel, einschließlich Soja, bezieht. Allerdings betrifft diese Deklarationspflicht nur abgepackte Ware, so dass z. B. lose Backwaren immer noch eine potenzielle Gefahr für Allergiker darstellen. Außerdem sollte überlegt werden, einen zusätzlichen Hinweis speziell für Birkenpollen- bzw. Sojaallergiker auf der Verpackung von Produkten mit hohem Gehalt an geringgradig prozessierten Sojaproteinen, z.B. auf den Verpackungen von Sojadrinks oder Sojadiätpulver, anzubringen. Dies wäre hilfreich, um potenziell lebensbedrohliche allergische Reaktionen zu vermeiden und andererseits bei stattgefundenen Reaktionen nachträglich den Betroffenen den Zusammenhang rascher deutlich zu machen.

\section{Therapie der Sojaallergie}

Allen Birkenpollenallergikern sollte im Sinne einer Primärprävention zur Vorsicht beim Verzehr von Sojaprodukten, insbesondere solcher mit hohem, nicht-fermentiertem Proteingehalt, geraten werden. Bei Patienten mit schwerer Soforttyp-Reaktion ist die Verordnung eines Notfallsets indiziert (Antihistaminika, Kortikosteroide, Adrenalin) [28].

Es gibt Hinweise darauf, dass sich unter einer spezifischen Immuntherapie (SIT) mit Baumpollenextrakten bei einem nennenswerten Anteil der Patienten auch die Pollen assoziierte Nahrungsmittelallergie (z.B. gegen Äpfel) bessern kann [29]. Entsprechende Untersuchungen u.a. mit Allergenextrakten mit 12,5-25 $\mu$ g Hauptallergengehalt durchgeführt [30]. Die begrenzten Effekte mancher dieser Studien wurden versucht damit zu erklären, dass für einen positiven Effekt auf die Nahrungsmit- telallergie die applizierte Allergendosis womöglich höher sein müsse als die zur Besserung der Pollen induzierten Atemwegsbeschwerden [30].

Insgesamt ist zu vermuten, dass eine SIT mit dem Hauptbirkenallergen Bet v 1 auch positive Effekte auf eine Gly m 4-bedingte Sojaallergie entfalten kann. Entsprechende kontrollierte Untersuchungen zur Überprüfung des Einsatzes eines hoch dosierten Bet v 1-Extrakts zur SIT im Rahmen der Birken assoziierten Sojaallergie wären sinnvoll.

\section{Abstract}

\section{Soy - an Underestimated Pollen Associated Food Allergen!? \\ $\nabla$}

Together with increased consumption of soy products there were increasing numbers of patients suffering from partly severe immediate type reactions, mainly after soy drinks, during the last years in Germany. Birch allergy has been identified as a special risk factor for developing such reactions. This is due to the high homology of the major allergens of birch (Bet v 1) and soy (Gly $\mathrm{m} 4$ ), which may lead to immunological cross reactivity. There are no exact data upon the prevalence of this type of pollen associated food allergy. Birch pollinosis is estimated to affect about $5-10 \%$ ( $4-8$ million) of the German population and up to 2.9 million might be at risk to suffer from a cross sensitization to soy. Allergen avoidance is complicated by the fact that soy may be a hidden food additive. A special warning of patients with birch pollinosis of consumption of non-fermented soy products with high protein content (i.e. drinks) seems to be justified. It is not known if specific immunotherapy against Bet $\mathrm{v} 1$ may improve symptoms of birch associated soy allergy.

\section{Literatur}

1 Treudler R, Werner M, Thiery J et al. High risk of immediate type reactions to soy drinks in 50 patients with birch pollinosis. J Investig Allergol Clin Immunol 2008; 18: 482 - 495

2 Süß A, Rytter M, Sticherling M, Simon JC. Anaphylaktische Reaktion auf ein Sojagetränk bei drei Patienten mit Birkenpollenallergie. JDDG 2005; 3: 895-897

3 Kleine-Tebbe J, Wangorsch A, Vogel L, Crowell DN, Haustein UF, Vieths S. Severe oral allergy syndrome and anaphylactic reactions caused by a Bet v 1- related PR-10 protein in soybean, SAM22. J Allerg Clin Immunol 2002; 110: 797-804

4 Kleine-Tebbe J, Herold DA, Vieths S. Sojaallergie durch Kreuzreaktionen gegen Birkenpollen-Majorallergen Bet v 1. Allergologie 2008; 31: $303-313$

5 Mateos-Aparicio I, Redondo Cuenca A, Villanueva-Suárez MJ, ZapataRevilla MA. Soybean, a promising health source. Nutr Hosp 2008; 23: $305-312$

6 Cross HS, Kállay E, Lechner D, Gerdenitsch W, Adlercreutz H, Armbrecht $H J$. Phytoestrogens and vitamin D metabolism: a new concept for the prevention and therapy of colorectal, prostate, and mammary carcinomas. J Nutr 2004; 134: 1207-1212

7 Averbeck M, Gebhardt C, Emmrich F, Treudler R, Simon JC. Immunologische Grundlagen der Allergien. J Dtsch Dermatol Ges 2007; 5: $1015-$ 28

8 Jäger L, Wüthrich B, Ballmer-Weber B, Vieths S. Nahrungsmittelallergien und -intoleranzen. 3. Auflage. München: Urban \& Fischer, Elsevier $\mathrm{GmbH}, 2008$ :

9 Hompes S, Kirschbaum J, Przybilla B et al. Anaphylaxis register in Central Europe: first results. Allergo J 2008; 17: 550-555

10 Treudler R, Kozovska Y, Simon JC. Severe immediate type hypersensitivity reactions in 105 German adults: When to diagnose anaphylaxis. J Invest Allergol Clin Immunol 2008; 18: $52-58$ 
11 Kleine-Tebbe J, Herold DA. Kreuzreaktive Allergenkluster bei pollenassoziierter Nahrungsmittelallergie. Hautarzt 2003; 54: 130-137

12 Ballmer-Weber BK. Kutane Symptome nach Genuss pollenassoziierter Nahrungsmittel. Hautarzt 2006; 47: 108-115

13 Cordle CT. Soy Protein Allergy: Incidence and relative severity. J Nutr 2004; 134: 1213 - 1219

14 Holzhauser T, Wackermann O, Ballmer-Weber BK et al. Soybean (Glycine max) allergy in Europe: Gly $\mathrm{m} 5$ (beta-conglycinin) and Gly $\mathrm{m}$ 6 (glycinin) are potential diagnostic markers for severe allergic reactions to soy. J Allergy Clin Immunol 2008; Nov 7 [Epub ahead of print]:

15 Batista R, Martins I, Jeno P, Ricardo CP, Oliveira MM. A proteomic study to identify soya allergens - the human response to transgenic versus non-transgenic soya samples. Int Arch Allergy Immunol 2007; 144: $29-38$

16 Ballmer-Weber BK, Vieths S. Soy allergy in perspective. Curr Opin Allergy Clin Immunol 2008; 8: 270-275

17 Anto JM, Sunyer J, Rodriguez-Roisin R, Suarez-Cervera M, Vazquez L. Community outbreaks of asthma associated with inhalation of soybean dust. Toxicoepidemiological Committee. N Engl J Med 1989; 320 : 1097-1102

18 Crowell DN, Maliyakal EJ, Russell D, Amasino RM. Characterization of a stress-induced, developmentally regulated gene family from soybean. Plant Molecular Biology 1992; 18: 459-466

19 Jahn-Schmid B, Radakovics A, Lüttkopf $D$ et al. Bet v $1_{142-156}$ is the dominant T-cell epitope of the major birch pollen allergen and important for cross-reactivity with Bet v 1-related food allergens. J Allergy Clin Immunol 2005; 11: $213-219$

20 Pedersen $M H$, Holzhauser T, Bisson $C$ et al. Soybean allergen detection methods - A comparison study. Mol Nutr Food Res 2008; 52 (12): $1486-1496$
21 Bruno G, Giampietro PG, Del GM, Gallia P, Giovannini L, Lovati C. Soy allergy is not common in atopic children: a multicenter study. Pediatr Allergy Immunol 1997; 8: 190-193

22 Kramer S, Gebhardt C, Averbeck $M$ et al. Anamnestische Soforttyp-Reaktionen bei fast jedem zweiten Birkenpollen-Allergiker nach Sojadrink-Verzehr. Allergo J 2008; 17: 65

23 Mittag D, Vieths S, Vogel L et al. Soybean allergy in patients allergic to birch pollen. Clinical investigation and molecular characterization of allergens. J Allergy Clin Immunol 2004; 113: 148-154

24 Hemmer W, Jung P, Sesztak-Greinecker G, Götz M. Birkenpollen-assoziierte Sojaallergie. Pädiatr Pädol 2008; 43: 17-20

25 Lidholm J, Ballmer-Weber BK, Mari A, Vieths S. Component-resolved diagnostics in food allergy. Curr Opin Allergy Clin Immunol 2006; 6: 234-240

26 Ballmer-Weber BK, Holzhauser T, Scibilia J et al. Clinical characteristics of soybean allergy in Europe: a double-blind, placebo-controlled food challenge study. J Allergy Clin Immunol 2007; 119: 1489-1496

27 Anibarro B, Seoane FJ, Mugica MV. Involvement of hidden allergens in food allergic reactions. J Invest Allergol Clin Immunol 2007; 17: 168 172

28 Ring J, Brockow K, Duda D et al. Akuttherapie anaphylaktischer Reaktionen - S2 Leitlinie. Allergo J 2007; 6: 420-434

29 Bolhaar STHP, Tiemessen MM, Zidmeer L et al. Efficacy of birch-pollen immunotherapy on cross-reactive food allergy confirmed by skin tests and double-blind food challenges. Clin Exp Allergy 2004; 34: $761-779$

30 Asero $R$. Effects of birch pollen SIT on apple allergy: a matter of dosage? Allergy 2004; 59: 1269-1271 